\title{
MAD FAMILIES, SPLITTING FAMILIES, AND LARGE CONTINUUM
}

\author{
JÖRG BRENDLE AND VERA FISCHER
}

\begin{abstract}
Let $\kappa<\lambda$ be regular uncountable cardinals. Using a finite support iteration of ccc posets we obtain the consistency of $\mathfrak{b}=\mathfrak{a}=\kappa<$ $\mathfrak{s}=\lambda$. If $\mu$ is a measurable cardinal and $\mu<\kappa<\lambda$, then using similar techniques we obtain the consistency of $\mathfrak{b}=\kappa<\mathfrak{a}=\mathfrak{s}=\lambda$.
\end{abstract}

\section{INTRODUCTION}

In the following we will study the bounding, splitting and almost disjointness numbers (for basic definitions and notation see [2]). Following standard notation ${ }^{\omega} \omega$ denotes the set of all functions from $\omega$ to $\omega$, $[\omega]^{\omega}$ denotes the set of infinite subsets of $\omega$ and $\leq^{*}$ denotes the eventual dominance order on ${ }^{\omega} \omega$. That is for $f, g$ in ${ }^{\omega} \omega f \leq^{*} g$ if and only if there is $n \in \omega$ such that for all $i \geq n(f(i) \leq g(i))$. A family $\mathcal{B} \subseteq \omega^{\omega} \omega$ is unbounded if there is no single real which simultaneously dominates all elements of $\mathcal{B}$. The bounding number $\mathfrak{b}$ is the minimal cardinality of an unbounded family. A family $\mathcal{A} \subseteq[\omega]^{\omega}$ is almost disjoint (a.d.) if any two distinct elements of $\mathcal{A}$ have finite intersection. An almost disjoint family $\mathcal{A}$ is maximal, called maximal almost disjoint, if for every $C \in[\omega]^{\omega}$ there is $A \in \mathcal{A}$ such that $|A \cap C|=\omega$. The almost disjointness number $\mathfrak{a}$ is the minimal cardinality of a maximal almost disjoint family. It is well known that $\mathfrak{b} \leq \mathfrak{a}$ (see [2]). A family $S \subseteq[\omega]^{\omega}$ is splitting if for every $A \in[\omega]^{\omega}$ there is $B \in S$ such that $|A \cap B|=\left|A \cap B^{c}\right|=\omega$. The splitting number $\mathfrak{s}$ is the minimal cardinality of a splitting family.

The bounding and the splitting numbers are independent. The consistency of $\mathfrak{s}<\mathfrak{b}$ was obtained in 1985 by J. Baumgartner and P. Dordal (see [1]). The consistency of $\mathfrak{b}<\mathfrak{s}$ was obtained in 1984 by S. Shelah (see [9]) using a proper forcing notion of size continuum, which is almost $\omega_{\omega} \omega$-bounding and adds a real not split by the ground model reals. There is an increased interest in obtaining models in which $\mathfrak{c} \geq \aleph_{3}$. In $1998 \mathrm{~J}$. Brendle obtained the consistency of $\mathfrak{b}=\kappa<\mathfrak{a}=\kappa^{+}$using a finite support iteration of ccc posets (see [5]). The consistency of $\mathfrak{b}=\kappa<\mathfrak{s}=\kappa^{+}$was obtained in [7] (see also [6]). In fact the forcing construction of the last two models, can be combined and in an appropriate finite support iteration of ccc posets one obtains the consistency of $\mathfrak{b}=\kappa<\mathfrak{a}=\mathfrak{s}=\kappa^{+}$.

The first author is partially supported by Grants-in-Aid for Scientific Research (C) 21540128 and (A) 19204008, Japan Society for the Promotion of Science, and by the Hausdorff Research Institute for Mathematics/Hausdorff Center for Mathematics, Universität Bonn.

The second author would like to thank the Austrian Science Fund FWF for postdoctoral support through grant no. P 20835-N13. 
In the present paper, we obtain the more general consistency results of $\mathfrak{b}=\mathfrak{a}=\kappa<\mathfrak{s}=\lambda$, for $\kappa, \lambda$ arbitrary regular uncountable cardinals (see Theorem 17) and $\mathfrak{b}=\kappa<\mathfrak{s}=\mathfrak{a}=\lambda$ where $\kappa<\lambda$ are arbitrary regular cardinals, above a measurable $\mu$ (see Theorem 21). Both of the constructions use the idea of matrix iteration introduced by A. Blass and S. Shelah in [3].

Notation: For an ultrafilter $\mathcal{U}$ on $\omega$, let $\mathbb{M}_{\mathcal{U}}$ denote the associated Mathias forcing (see [2]). That is $\mathbb{M}_{\mathcal{U}}$ is the poset of all $(a, A) \in[\omega]^{<\omega} \times \mathcal{U}$ such that $\max a<\min A$ with extension relation defined as follows: $\left(a_{1}, A_{1}\right) \leq\left(a_{2}, A_{2}\right)$ if $a_{2}$ is an initial segment of $a_{1}, a_{1} \backslash a_{2} \subseteq A_{2}$ and $A_{1} \subseteq A_{2}$. The Hechler forcing $\mathbb{D}$ (see [2]) consists of all $(s, f) \in<\omega_{\omega} \times{ }^{\omega} \omega$ with extension relation defined as follows: $\left(s_{1}, f_{1}\right) \leq\left(s_{2}, f_{2}\right)$ if $s_{2}$ is an initial segment of $s_{1}$, for all $i \in \operatorname{dom}\left(s_{1}\right) \backslash \operatorname{dom}\left(s_{2}\right), s_{1}(i) \geq f_{2}(i)$ and for all $i \in \omega f_{2}(i) \leq f_{1}(i)$. For $\mu$ a measurable cardinal and $\mathcal{D}$ a $\mu$-complete ultrafilter on $\mu$, let $\mathbb{P}^{\mu} / \mathcal{D}$ denote the ultrapower of $\mathbb{P}$ (see [4]), where $\mathbb{P}$ is a given poset. Ultrapowers of posets were introduced by S. Shelah in [10].

\section{AdDING A MAD FAMILY}

Definition 1 (S. Hechler [8]). For $\gamma$ an ordinal, $\mathbb{P}_{\gamma}$ is the poset of all finite partial functions $p: \gamma \times \omega \rightarrow 2$ such that $\operatorname{dom}(p)=F_{p} \times n_{p}$ where $F_{p} \in[\gamma]^{<\omega}$, $n_{p} \in \omega$. The order is given by $q \leq p$ if $p \subseteq q$ and $\left|q^{-1}(1) \cap F^{p} \times\{i\}\right| \leq 1$ for all $i \in n_{q} \backslash n_{p}$.

$\mathbb{P}_{\gamma}$ is ccc. If $G$ is $\mathbb{P}_{\gamma}$-generic, then the family $\mathcal{A}_{\gamma}=\left\{A_{\alpha}: \alpha<\gamma\right\}$, where $A_{\alpha}=\{i: \exists p \in G p(\alpha, i)=1\}$ is almost disjoint and for $\gamma \geq \omega_{1}$ maximal almost disjoint (see [8]). This product like forcing decomposes as a two-step iteration as follows. Let $\gamma<\delta, G$ a $\mathbb{P}_{\gamma}$-generic filter. In $V[G]$, let $\mathbb{P}_{[\gamma, \delta)}$ be the poset of all pairs $(p, H)$ such that $p:(\delta \backslash \gamma) \times \omega \rightarrow 2$ is a finite partial function with $\operatorname{dom}(p)=F_{p} \times n_{p}$ where $F_{p} \in[\delta \backslash \gamma]^{<\omega}, n_{p} \in \omega$ and $H \in[\gamma]^{<\omega}$. The order is given by $(q, K) \leq(p, H)$ if $q \leq_{\mathbb{P}_{\delta}} p, H \subseteq K$ and for all $\alpha \in F_{p}$, $\beta \in H, i \in n_{q} \backslash n_{p}$ if $i \in A_{\beta}$, then $q(\alpha, i)=0$. Observe that $\mathbb{P}_{\delta}=\mathbb{P}_{\gamma} * \dot{\mathbb{P}}_{[\gamma, \delta)}$, i.e. $\mathbb{P}_{\delta}$ is forcing equivalent to the two step iteration of $\mathbb{P}_{\gamma}$ and $\dot{\mathbb{P}}_{[\gamma, \delta)}$.

Definition 2. Let $M \subseteq N$ be models of set theory, $\mathcal{B}=\left\{B_{\alpha}\right\}_{\alpha<\gamma} \subseteq M \cap[\omega]^{\omega}$, $A \in N \cap[\omega]^{\omega}$. Then $\left(\star_{\mathcal{B}, A}^{M}\right)$ holds if for every $h: \omega \times[\gamma]^{<\omega} \rightarrow \omega, h \in M$ and $m \in \omega$ there are $n \geq m, F \in[\gamma]^{<\omega}$ such that $[n, h(n, F)) \backslash \bigcup_{\alpha \in F} B_{\alpha} \subseteq A$.

Lemma 3. Let $\left(\star_{\mathcal{B}, A}^{M, N}\right)$ hold, $\mathcal{B}=\left\{B_{\alpha}\right\}_{\alpha<\gamma}$, let $\mathcal{I}(\mathcal{B})$ be the ideal generated by $\mathcal{B}$ and the finite sets and let $B \in M \cap[\omega]^{\omega}, B \notin \mathcal{I}(\mathcal{B})$. Then $|A \cap B|=\aleph_{0}$.

Proof. Otherwise $A \cap B \subseteq n$ for some $n \in \omega$. Let $m \geq n, F \in[\gamma]<\omega$. Since $B \notin \mathcal{I}(\mathcal{B}), B \nsubseteq \complement^{*} \bigcup_{\alpha \in F} B_{\alpha}$ and so there is $k_{m, F} \in B \backslash \bigcup_{\alpha \in F} B_{\alpha}$ greater than $m$. Define $h(m, F)=k_{m, F}+1$ for all $m \geq n, F \in[\gamma]^{<\omega}$ and $h\left\lceil n \times[\gamma]^{<\omega}=0\right.$. Then $h$ is a function in $M$ such that $[m, h(m, F)) \backslash \bigcup_{\alpha \in F} B_{\alpha} \nsubseteq A$ for all $m \geq n, F \in[\gamma]^{<\omega}$, contradicting $\left(\star_{\mathcal{B}, A}^{M, N}\right)$.

The sets $A_{\alpha}$ added by the forcing $\mathbb{P}_{\gamma}$ satisfy the above property in the following sense:

Lemma 4. Let $G_{\gamma+1}$ be $\mathbb{P}_{\gamma+1}$-generic, $G_{\gamma}=G_{\gamma+1} \cap \mathbb{P}_{\gamma}$ and $\mathcal{A}_{\gamma}=\left\{A_{\alpha}\right\}_{\alpha<\gamma}$, where $A_{\alpha}=\left\{i: \exists p \in G_{\gamma+1} p(\alpha, i)=1\right\}, \alpha \leq \gamma$. Then $\left(\star_{\mathcal{A}_{\gamma}, A_{\gamma}\left[G_{\gamma}\right], V\left[G_{\gamma+1}\right]}\right)$ holds. 
Proof. Let $h \in V\left[G_{\gamma}\right], h: \omega \times[\gamma]^{<\omega} \rightarrow \omega,(p, H) \in \mathbb{P}_{[\gamma, \gamma+1)}, m \in \omega$. Then $\operatorname{dom}(p)=\{\gamma\} \times n_{p}$ where $n_{p} \in \omega$. Define an extension $(q, K)$ of $(p, H)$ in $\mathbb{P}_{\{\gamma, \gamma+1)}$ as follows. Let $n>\max \left\{m, n_{p}\right\}, n_{q}=h(n, H)$. Let $\operatorname{dom}(q)=\{\gamma\} \times n_{q}, K=H, q\left\lceil\{\gamma\} \times n_{p}=p, q\left\lceil\{\gamma\} \times\left[n_{p}, n\right)=0\right.\right.$ and for $i \in\left[n, n_{q}\right)$ let $q(\gamma, i)=1$ if and only if $i \notin \bigcup_{\alpha \in H} A_{\alpha}$. Then $(q, K) \leq(p, H)$ and $(q, K) \Vdash[n, h(n, H)) \backslash \bigcup_{\alpha \in H} A_{\alpha} \subseteq A_{\gamma}$.

\section{Combinatorics And PReservation}

In addition to $\left(\star_{\mathcal{B}, A}^{M, N}\right)$, we consider one more combinatorial property which will be crucial for the second consistency result to be established, and systemize some preservation theorems for both of these properties.

Definition 5. If $M \subseteq N$ are models of set theory, $c \in N \cap[\omega]^{\omega}$ such that for all $f \in M \cap[\omega]^{\omega}, N \vDash c \mathbb{Z}^{*} f$, we will say that $(\star M, N, c)$ holds.

The following lemma can be found in [3].

Lemma 6 (A. Blass, S. Shelah, [3]). Let $M \subseteq N$ be models of set theory, $\mathcal{U}$ an ultrafilter in $M, c \in \omega^{\omega} \cap N$ such that $(\star M, N, c)$ holds. Then there is an ultrafilter $\mathcal{V} \supseteq \mathcal{U}$ in $N$ such that:

(1) every maximal antichain of $\mathbb{M}_{\mathcal{U}}$ which belongs to $M$ is a maximal antichain of $\mathbb{M}_{\mathcal{V}}$ in $N$,

(2) $(\star M[G], N[G], c)$ holds where $G$ is $\mathbb{M}_{\mathcal{V}}$-generic over $N$ (and thus, by

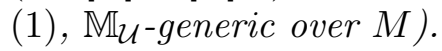

In analogy, we obtain the following.

Crucial Lemma 7. Let $M \subseteq N$ be models of set theory, $\mathcal{B}=\left\{B_{\alpha}\right\}_{\alpha<\gamma} \subseteq$ $M \cap[\omega]^{\omega}, A \in N \cap[\omega]^{\omega}$ such that $\left(\star_{\mathcal{B}, A}^{M, N}\right)$ holds. Let $\mathcal{U}$ be an ultrafilter in $M$. Then there is an ultrafilter $\mathcal{V} \supseteq \mathcal{U}$ in $N$ such that

(1) every maximal antichain of $\mathbb{M}_{\mathcal{U}}$ which belongs to $M$ is a maximal antichain of $\mathbb{M}_{\mathcal{V}}$ in $N$,

(2) $\left(\star_{\mathcal{B}, A}^{M[G], N[G]}\right)$ holds where $G$ is $\mathbb{M}_{\mathcal{V}}$-generic over $N$ (and thus, by (1), $\mathbb{M}_{\mathcal{U}}$-generic over $\left.M\right)$.

Proof. Work in $N$. Let $C \subseteq \mathbb{M}_{\mathcal{U}}, C \in M$, be a maximal antichain, and let $s \in[\omega]^{<\omega}$. We say $X$ is forbidden by $C, s$ if $(s, X)$ is incompatible with all conditions from $C$.

Given an $\mathbb{M}_{\mathcal{U}}$-name $\dot{h}: \omega \times[\gamma]^{<\omega} \rightarrow \omega, \dot{h} \in M$, there are (in $M$ ) maximal antichains $D_{n, F}^{\dot{h}} \subseteq \mathbb{M}_{\mathcal{U}}$ and functions $g_{n, F}^{\dot{h}}: D_{n, F}^{\dot{h}} \rightarrow \omega$ such that $p$ forces that $\dot{h}(n, F)=g_{n, F}^{\dot{h}}(p)$ for all $p \in D_{n, F}^{\dot{h}}$. Say $Y$ is forbidden by $\dot{h}, t$ if, for all $n$ and all $F,(t, Y)$ is incompatible with all conditions $p \in D_{n, F}^{\dot{h}}$ which satisfy $\left[n, g_{n, F}^{\dot{h}}(p)\right) \backslash \bigcup_{\alpha \in F} B_{\alpha} \subseteq A$. (This means that $(t, Y)$ forces that $[n, \dot{h}(n, F)) \backslash \bigcup_{\alpha \in F} B_{\alpha} \not A$ for all $n, F$.)

Let $\mathcal{I}$ be the ideal generated by all forbidden sets.

Main Claim 8. $\mathcal{I} \cap \mathcal{U}=\emptyset$

Once the main claim in proved, we construct $\mathcal{V} \supseteq \mathcal{U}$ such that $\mathcal{V} \cap \mathcal{I}=\emptyset$. Then (1) and (2) easily hold. 
Proof. By contradiction. Assume there are forbidden sets

$$
X_{0}, \ldots, X_{k-1}, Y_{0}, \ldots, Y_{k-1}
$$

with witnesses

$$
C_{0}, s_{0}, \ldots, C_{k-1}, s_{k-1}, \dot{h}_{0}, t_{0}, \ldots, \dot{h}_{k-1}, t_{k-1}
$$

such that $Z:=\bigcup_{i<k} X_{i} \cup \bigcup_{i<k} Y_{i} \in \mathcal{U}$ (in $M$ ).

For $t \in[\omega]^{<\omega}$ and $(s, X) \in \mathbb{M}_{\mathcal{U}}$, say $(s, X)$ permits $t$ if $s \subseteq t \subseteq s \cup X$. If $C \subseteq \mathbb{M}_{\mathcal{U}}$, say $C$ permits $t$ if there is $p \in C$ which permits $t$. Note that $p$ is compatible with $(t, Y)$ iff there is $u \subseteq Y$ such that $p$ permits $t \cup u$.

Subclaim 9. There is $h: \omega \times[\gamma]^{<\omega} \rightarrow \omega, h \in M$, with $h(n, F) \geq n$, such that whenever we partition $Z \cap[n, h(n, F))$ into $2 k$ pieces, then at least one piece $s$ has the following property:

- for all $i<k$ there is $t \subseteq s$ such that $C_{i}$ permits $s_{i} \cup t$,

- for all $i<k$ there is $t \subseteq s$ such that some $p \in D_{n, F}^{\dot{h}_{i}}$ with $g_{n, F}^{\dot{h}_{i}}(p)<$ $h(n, F)$ permits $t_{i} \cup t$.

Proof. The subclaim only mentions objects from $M$, and is clearly absolute. Therefore we may prove it in $M$.

Assume the subclaim was false for some $n$ and $F$. Consider $Z \backslash n$. By a compactness argument (equivalently, by König's Lemma) we could partition $Z \backslash n$ into $2 k$ pieces none of which satisfies the conclusion of the subclaim. One of the $2 k$ pieces, say $W$, must belong to $\mathcal{U}$. Since $C_{i}$ is a maximal antichain, there is $p \in C_{i}$ such that $p$ and $\left(s_{i}, W\right)$ are compatible. Thus there is $t \subseteq W$ such that $p$ permits $s_{i} \cup t$. Similarly, there are $p \in D_{n, F}^{\dot{h}_{i}}$ and $t \subseteq W$ such that $p$ permits $t_{i} \cup t$. If we choose $h(n, F)$ large enough, $s=$ $W \cap[n, h(n, F))$ has the required properties, contradictory to our assumption about $n$ and $F$.

We continue the proof of the main claim. Fix $n$ and $F$. Consider the partition given by $\left\{X_{i} \cap[n, h(n, F)), Y_{i} \cap[n, h(n, F)): i<k\right\}$. Consider a piece $X_{i} \cap[n, h(n, F))$. Since $\left(s_{i}, X_{i}\right)$ is incompatible with all conditions from $C_{i}$, there is no $t \subseteq X_{i} \cap[n, h(n, F))$ such that $C_{i}$ permits $s_{i} \cup t$. So $X_{i} \cap$ $[n, h(n, F))$ is not as in the subclaim. Hence one piece $s=Y_{i} \cap[n, h(n, F))$ satisfies the conclusion of the subclaim. Thus there are $t \subseteq Y_{i} \cap[n, h(n, F))$ and $p \in D_{n, F}^{\dot{h}_{i}}$ with $g_{n, F}^{\dot{h}_{i}}(p)<h(n, F)$ such that $p$ permits $t_{i} \cup t$. In particular $p$ is compatible with $\left(t_{i}, Y_{i}\right)$. On the other hand, $\left(t_{i}, Y_{i}\right)$ is incompatible with all $q \in D_{n, F}^{\dot{h}_{i}}$ which satisfy $\left[n, g_{n, F}^{\dot{h}_{i}}(q)\right) \backslash \bigcup_{\alpha \in F} B_{\alpha} \subseteq A$. Thus $\left[n, g_{n, F}^{\dot{h}_{i}}(p)\right) \backslash$ $\bigcup_{\alpha \in F} B_{\alpha} \not \subseteq A$, and $[n, h(n, F)) \backslash \bigcup_{\alpha \in F} B_{\alpha} \not \nsubseteq A$ follows. Unfixing $n$ and $F$, we see this holds for all $n$ and $F$. This contradicts $\left(\star_{\mathcal{B}, A}^{M, N}\right)$, and the proof of the main claim and the crucial lemma is complete.

Lemma 10. Let $\left\langle\mathbb{P}_{\ell, \eta}, \dot{\mathbb{Q}}_{\ell, \eta}: \eta<\zeta\right\rangle$, $\ell \in\{0,1\}$ be finite support iterations such that $\mathbb{P}_{0, \eta}$ is a complete suborder of $\mathbb{P}_{1, \eta}$ for all $\eta<\zeta$. Then $\mathbb{P}_{0, \zeta}$ is a complete suborder of $\mathbb{P}_{1, \zeta}$. 
Proof. It is clear that $\mathbb{P}_{0, \zeta} \subseteq \mathbb{P}_{1, \zeta}$ and that incompatibility is preserved. Let $p \in \mathbb{P}_{1, \zeta}$. Since $\mathbb{P}_{1, \zeta}$ is finite support iteration, there is $\eta<\zeta$ such that $p \in \mathbb{P}_{1, \eta}$. However $\mathbb{P}_{0, \eta}<0 \mathbb{P}_{1, \eta}$ and so there is $q \in \mathbb{P}_{0, \eta}$ which is a reduction of $p$ (in $\mathbb{P}_{0, \eta}$ ). Then $q$ is also a reduction of $p$ in $\mathbb{P}_{0, \zeta}$. Indeed, let $r \leq q$, $r \in \mathbb{P}_{0, \zeta}$. Then $r=r_{0} \cup r_{1}$ where $r_{0} \in \mathbb{P}_{0, \eta}$ and $\operatorname{suppt}\left(r_{1}\right) \subseteq[\eta, \zeta)$. Then $r_{0} \leq q$ and since $q$ is a reduction of $p$ in $\mathbb{P}_{0, \eta}$, there is $\tilde{r}_{0} \in \mathbb{P}_{0, \eta}$ which is a common extension of $p$ and $r_{0}$. Then $\tilde{r}_{0} \cup r_{1}$ is a common extension of $r$ and $p$. Thus $q$ is a reduction of $p$ in $\mathbb{P}_{0, \zeta}$.

Lemma 11. Let $M \subseteq N$ be models of set theory, $\mathbb{P} \in M$ a poset such that $\mathbb{P} \subseteq M, G$ a $\mathbb{P}$-generic filter over $M$ (and so $\mathbb{P}$-generic over $N$ ).

(1) Let $\mathcal{B}=\left\{B_{\alpha}\right\}_{\alpha \in \gamma} \subseteq M \cap[\omega]^{\omega}, A \in N \cap[\omega]^{\omega}$ such that $\left(\star_{\mathcal{B}, A}^{M, N}\right)$ holds. Then $\left(\star_{\mathcal{B}, A}^{M[G], N[G]}\right)$ holds.

(2) Let $c \in N \cap{ }^{\omega} \omega$ such that $(\star M, N, c)$. Then $(\star M[G], N[G], c)$ holds.

Proof. We give a proof only of (1), since part (2) is proved similarly. If (1) does not hold, then there are $h \in M[G], h: \omega \times[\gamma]^{<\omega} \rightarrow \omega$ and $m \in \omega$ such that for all $n \geq m, F \in[\gamma]^{<\omega}, N[G] \vDash[n, h(n, F)) \backslash \bigcup_{\alpha \in F} B_{\alpha} \not \subset A$. Then there are a $\mathbb{P}$-name $\dot{h}$ for $h$ in $M, p \in G$ and $m \in \omega$ such that

$$
p \Vdash_{N, \mathbb{P}} \forall n \geq m \forall F \in[\gamma]^{<\omega}\left([n, \dot{h}(n, F)) \backslash \bigcup_{\alpha \in F} B_{\alpha} \not \subset A\right) .
$$

However for all $n \geq m, F \in[\gamma]^{<\omega}$ there are $p_{n, F} \leq p$ (in $M$ ) and $k_{n, F} \in \omega$ such that $p_{n, F} \Vdash_{M, \mathbb{P}} \dot{h}(n, F)=k_{n, F}$. Then

$$
p_{n, F} \Vdash_{N, \mathbb{P}}\left[n, k_{n, F}\right) \backslash \bigcup_{\alpha \in F} B_{\alpha} \not \subset A
$$

and so $N \vDash\left[n, k_{n, F}\right) \backslash \bigcup_{\alpha \in F} B_{\alpha} \nsubseteq A$. In $M$ define $h_{0}: \omega \times[\gamma]^{<\omega} \rightarrow \omega$ as follows. Let $h_{0}\left\lceil m \times[\gamma]^{<\omega}=0\right.$ and for all $n \geq m, F \in[\gamma]^{<\omega}$ let $h_{0}(n, F)=$ $k_{n, F}$. Then $h_{0}$ gives a contradiction to $\left(\star_{\mathcal{B}, A}^{M, N}\right)$.

Lemma 12. Let $\left\langle\mathbb{P}_{\ell, n}, \dot{\mathbb{Q}}_{\ell, n}: n \in \omega\right\rangle, \ell \in\{0,1\}$ be finite support iterations such that $\mathbb{P}_{0, n}$ is a complete suborder of $\mathbb{P}_{1, n}$ for all $n$. Let $V_{\ell, n}=V^{\mathbb{P}_{\ell, n}}$.

(1) Let $\mathcal{B}=\left\{A_{\gamma}\right\}_{\gamma<\alpha} \subseteq V_{0,0} \cap[\omega]^{\omega}, A \in V_{1,0} \cap[\omega]^{\omega}$. If $\left(\star_{\mathcal{B}, n}^{V_{0, n}, V_{1, n}}\right)$ holds for all $n \in \omega$, then $\left(\star_{\mathcal{B}, A}^{V_{0,}, V_{1, \omega}}\right)$ holds.

(2) Let $c \in V_{1,0} \cap{ }^{\omega} \omega$. If $\left(\star V_{0, n}, V_{1, n}, c\right)$ holds for all $n \in \omega$, then $\left(\star V_{0, \omega}, V_{1, \omega}, c\right)$ holds.

Proof. We will give a proof of (1). The proof of (2) is analogous. Thus suppose the claim of (1) does not hold and let $h: \omega \times[\alpha]^{<\omega} \rightarrow \omega$ be a function in $V_{0, \omega}$ such that for some $m \in \omega$, for all $n \geq m, F \in[\alpha]^{<\omega}$, $V_{1, \omega} \vDash[n, h(n, F)) \backslash \bigcup_{\gamma \in F} A_{\gamma} \not A$. Then there are a $\mathbb{P}_{0, \omega}$-name $\dot{h}, p \in \mathbb{P}_{1, \omega}$ such that $p \Vdash[k, \dot{h}(k, F)) \backslash \bigcup_{\gamma \in F} A_{\gamma} \nsubseteq A$ for all $k \geq m, F \in[\alpha]^{<\omega}$. Since $p$ has finite support, there is $n \in \omega$ such that $p \in \mathbb{P}_{1, n}$. Let $G_{1, n}$ be a $\mathbb{P}_{1, n}$-generic filter containing $p$ and let $h^{\prime}=\dot{h} / G_{0, n}$ be the quotient name, where $G_{0, n}=G_{1, n} \cap \mathbb{P}_{0, n}$. Let $\mathbb{R}_{n, \omega}^{\ell}$ be the quotient poset $\mathbb{P}_{\ell, n} / G_{\ell, n}$ in 
$V_{\ell, n}=V\left[G_{\ell, n}\right]$. Then $h^{\prime} \in V_{0, n}$ and for all $k \geq m, F \in[\alpha]^{<\omega}$

$$
V_{n, 1} \vDash \Vdash_{\mathbb{R}_{n, \omega}^{1}}\left[k, h^{\prime}(k, F)\right) \backslash \bigcup_{\gamma \in F} A_{\gamma} \nsubseteq \AA .
$$

Then for all $k \geq m, F \in[\alpha]^{<\omega}$ find $p_{k, F} \in \mathbb{R}_{n, \omega}^{0}$ and $x_{k, F} \in \omega$ such that $p_{k, F} \Vdash h^{\prime}(k, F)=x_{k, F}$ and define $h_{0}(k, F)=x_{k, F}$. Let $h_{0}\left\lceil m \times[\alpha]^{<\omega}=0\right.$. Then $h_{0} \in V_{0, n}$ and $\left[k, h_{0}(k, F)\right) \backslash \bigcup_{\gamma \in F} A_{\gamma} \not \subset A$ for all $k \geq m, F \in[\alpha]^{<\omega}$ contradicting $\left(\star_{\mathcal{B}, A}^{V_{0, n}, V_{1, n}}\right)$.

The following Lemma is well-known and often used.

Lemma 13. Let $\mathbb{P}, \mathbb{Q}$ be partial orders, such that $\mathbb{P}$ is completely embedded into $\mathbb{Q}$. Let $\dot{\mathbb{A}}$ be a $\mathbb{P}$-name for a forcing notion, $\dot{\mathbb{B}}$ a $\mathbb{Q}$-name for a forcing notion such that $\vdash_{\mathbb{Q}} \dot{\mathbb{A}} \subseteq \dot{\mathbb{B}}$, and every maximal antichain of $\dot{\mathbb{A}}$ in $V^{\mathbb{P}}$ is a maximal antichain of $\dot{\mathbb{B}}$ in $V^{\mathbb{Q}}$. Then $\mathbb{P} * \dot{\mathbb{A}}<0 \mathbb{Q} * \dot{\mathbb{B}}$.

Proof. It is sufficient to show that every maximal antichain of $\mathbb{P} * \dot{\mathbb{A}}$ is a maximal antichain of $\mathbb{Q} * \dot{\mathbb{B}}$. Thus let $\left\{\left(p_{\alpha}, \dot{a}_{\alpha}\right): \alpha<\kappa\right\}$ be a maximal antichain of $\mathbb{P} * \dot{\mathbb{A}}$. Suppose it is not maximal in $\mathbb{Q} * \dot{\mathbb{B}}$ and let $(q, \dot{b})$ be a condition in $\mathbb{Q} * \dot{\mathbb{B}}$ which is incompatible with all $\left(p_{\alpha}, \dot{a}_{\alpha}\right)$ for $\alpha<\kappa$. Let $\dot{H}$ be the canonical $\mathbb{P}$-name for the $\mathbb{P}$-generic filter and let $\dot{\Omega}$ be a $\mathbb{P}$-name such that $\Vdash \dot{\Omega}=\left\{\alpha: p_{\alpha} \in \dot{H}\right\}$.

Claim. $\Vdash "\left\{\dot{a}_{\alpha}: \alpha \in \dot{\Omega}\right\}$ is a maximal antichain of $\dot{\mathbb{A}} "$.

Proof. Suppose not. Then, there are $p \in \mathbb{P}$ and a $\mathbb{P}$-name $\dot{a}$ such that $p \Vdash \forall \alpha\left(\alpha \in \dot{\Omega} \rightarrow \dot{a} \perp \dot{a}_{\alpha}\right)$. Then $(p, \dot{a}) \in \mathbb{P} * \dot{\mathbb{A}}$ and so there is $\alpha<\kappa$ such that $(p, \dot{a}) \not \perp\left(p_{\alpha}, \dot{a}_{\alpha}\right)$. Let $\left(p^{\prime}, \dot{a}^{\prime}\right)$ be a common extension. Then $p^{\prime} \Vdash\left(\dot{a}^{\prime} \leq \dot{a}\right.$ and $\left.\dot{a}^{\prime} \leq \dot{a}_{\alpha}\right)$, and since $p^{\prime} \leq p_{\alpha}, p^{\prime} \Vdash \alpha \in \dot{\Omega}$. That is, $p^{\prime} \Vdash\left(\alpha \in \dot{\Omega}\right.$ and $\left.\dot{a}^{\prime} \leq \dot{a}, \dot{a}^{\prime} \leq \dot{a}_{\alpha}\right)$ which is a contradiction.

Let $G$ be $\mathbb{Q}$-generic filter such that $q \in G$. Then since $\mathbb{P}<0 \mathbb{Q}$, there is a $\mathbb{P}$-generic filter $H$ such that $V[H] \subseteq V[G]$. Now let $b=\dot{b}[G], a_{\alpha}=\dot{a}_{\alpha}[G]=$ $\dot{a}_{\alpha}[H]$ (for $\alpha$ such that $p_{\alpha} \in H$ ) and let $\Omega=\dot{\Omega}[G]=\left\{\alpha<\kappa: p_{\alpha} \in H\right\}$. By the above claim $\left\{a_{\alpha}: \alpha \in \Omega\right\}$ is a maximal antichain in $\mathbb{A}$ (in $V[G]$ ) and so by the hypothesis of the Lemma, it is a maximal antichain of $\mathbb{B}$ (in $V[G]$ ). So there is $\alpha \in \Omega$ such that $b=\dot{b}[G]$ is compatible with $a_{\alpha}=\dot{a}_{\alpha}[H]$. So there is $q^{\prime} \in G$ such that $q^{\prime} \leq p_{\alpha}, q^{\prime} \leq q$ and $q^{\prime} \Vdash\left(\alpha \in \dot{\Omega}\right.$ and $\left.\dot{b} \not \perp \dot{a}_{\alpha}\right)$. Thus there is a $\mathbb{Q}$-name $\dot{b}^{\prime}$ such that $q^{\prime} \Vdash " \dot{b}^{\prime} \leq \dot{b}, \dot{b}^{\prime} \leq \dot{a}_{\alpha}$ " and so $\left(q^{\prime}, \dot{b}^{\prime}\right)$ is a common extension of $(q, \dot{b})$ and $\left(p_{\alpha}, \dot{a}_{\alpha}\right)$, which is a contradiction.

\section{The COnsistency OF $\mathfrak{b}=\mathfrak{a}=\kappa<\mathfrak{s}=\lambda$}

Let $f:\{\eta<\lambda: \eta \equiv 1 \bmod 2\} \rightarrow \kappa$ be an onto mapping, such that for all $\alpha<\kappa, f^{-1}(\alpha)$ is cofinal in $\lambda$. Recursively define a system of finite support iterations $\left\langle\left\langle\mathbb{P}_{\alpha, \zeta}: \alpha \leq \kappa, \zeta \leq \lambda\right\rangle,\left\langle\dot{\mathbb{Q}}_{\alpha, \zeta}: \alpha \leq \kappa, \zeta<\lambda\right\rangle\right\rangle$ as follows. For all $\alpha, \zeta$ let $V_{\alpha, \zeta}=V^{\mathbb{P}_{\alpha, \zeta}}$. We refer to such systems as matrix iterations. Note that this type of iterations appeared for the first time in [3].

(1) If $\zeta=0$, then for all $\alpha \leq \kappa, \mathbb{P}_{\alpha, 0}$ is Hechler's poset (see Definition 1) for adding an a.d. family $\mathcal{A}_{\alpha}=\left\{A_{\beta}\right\}_{\beta<\alpha}$ (note that for $\alpha \geq \omega_{1}, \mathcal{A}_{\alpha}$ is mad in $\left.V_{\alpha, 0}\right)$. 
(2) If $\zeta=\eta+1, \zeta \equiv 1 \bmod 2$, then $\Vdash_{\mathbb{P}_{\alpha, \eta}} \dot{\mathbb{Q}}_{\alpha, \eta}=\mathbb{M}_{\dot{\mathcal{U}}_{\alpha, \eta}}$ where $\dot{\mathcal{U}}_{\alpha, \eta}$ is a $\mathbb{P}_{\alpha, \eta}$-name for an ultrafilter and for all $\alpha<\beta \leq \kappa, \Vdash_{\mathbb{P}_{\beta, \eta}} \dot{\mathcal{U}}_{\alpha, \eta} \subseteq \dot{\mathcal{U}}_{\beta, \eta}$.

(3) If $\zeta=\eta+1, \zeta \equiv 0 \bmod 2$, then if $\alpha \leq f(\eta), \dot{\mathbb{Q}}_{\alpha, \eta}$ is a $\mathbb{P}_{\alpha, \eta}$-name for the trivial forcing notion; if $\alpha>f(\eta)$ then $\dot{\mathbb{Q}}_{\alpha, \eta}$ is a $\mathbb{P}_{\alpha, \eta}$-name for $\mathbb{D}^{V_{f(\eta), \eta}}$.

(4) If $\zeta$ is a limit, then for all $\alpha \leq \kappa, \mathbb{P}_{\alpha, \zeta}$ is the finite support iteration of $\left\langle\mathbb{P}_{\alpha, \eta}, \dot{\mathbb{Q}}_{\alpha, \eta}: \eta<\zeta\right\rangle$.

Furthermore the construction will satisfy the following two properties:

(a) $\forall \zeta \leq \lambda \forall \alpha<\beta \leq \kappa, \mathbb{P}_{\alpha, \zeta}$ is a complete suborder of $\mathbb{P}_{\beta, \zeta}$.

(b) $\forall \zeta \leq \lambda \forall \alpha<\kappa\left(\star_{\mathcal{A}_{\alpha}, A_{\alpha}}^{V_{\alpha, \zeta}, V_{\alpha+1, \zeta}}\right)$ holds.

Proceed by recursion on $\zeta$. For $\zeta=0, \alpha \leq \kappa$ let $\mathbb{P}_{\alpha, 0}$ be the poset from Definition 1. Then by the product property of $\mathbb{P}_{\alpha, 0}$ and Lemma 4 respectively, properties $(a)$ and $(b)$ above hold. Let $\zeta=\eta+1$ be a successor ordinal and suppose $\forall \alpha \leq \kappa, \mathbb{P}_{\alpha, \eta}$ has been defined so that properties $(a)$ and $(b)$ above hold.

If $\zeta \equiv 1 \bmod 2$ define $\dot{\mathbb{Q}}_{\alpha, \eta}$ by induction on $\alpha \leq \kappa$ as follows. If $\alpha=0$, let $\dot{\mathcal{U}}_{0, \eta}$ be a $\mathbb{P}_{0, \eta}$-name for an ultrafilter, $\dot{\mathbb{Q}}_{0, \eta}$ a $\mathbb{P}_{0, \eta}$-name for $\mathbb{M}_{\dot{\mathcal{U}}_{0, \eta}}$ and let $\mathbb{P}_{0, \zeta}=\mathbb{P}_{0, \eta} * \dot{\mathbb{Q}}_{0, \eta}$. If $\alpha=\beta+1$ and $\dot{\mathcal{U}}_{\beta, \eta}$ has been defined, by the inductive hypothesis and Lemma 7 there is a $\mathbb{P}_{\alpha, \eta}$-name $\dot{\mathcal{U}}_{\alpha, \eta}$ for an ultrafilter such that $\Vdash_{\mathbb{P}_{\alpha, \eta}} \dot{\mathcal{U}}_{\beta, \eta} \subseteq \dot{\mathcal{U}}_{\alpha, \eta}$, every maximal antichain of $\mathbb{M}_{\dot{\mathcal{U}}_{\beta, \eta}}$ in $V_{\beta, \eta}$ is a

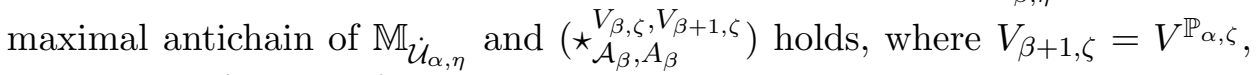
$\mathbb{P}_{\alpha, \zeta}=\mathbb{P}_{\alpha, \eta} * \dot{\mathbb{Q}}_{\alpha, \eta}$ and $\dot{\mathbb{Q}}_{\alpha, \eta}$ is a $\mathbb{P}_{\alpha, \eta}$-name for $\mathbb{M}_{\dot{\mathcal{U}}_{\alpha, \eta}}$. Note that by Lemma 13 $\mathbb{P}_{\beta, \zeta}=\mathbb{P}_{\beta, \eta} * \mathbb{M}_{\dot{\mathcal{U}}_{\beta, \eta}}$ is a complete suborder of $\mathbb{P}_{\alpha, \zeta}$. If $\alpha$ is a limit ordinal and

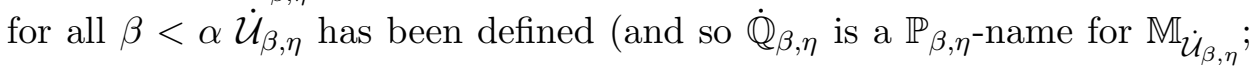
$\left.\mathbb{P}_{\beta, \zeta}=\mathbb{P}_{\beta, \eta} * \dot{\mathbb{Q}}_{\beta, \eta}\right)$, consider the following two cases. If $\operatorname{cf}(\alpha)=\omega$, find a $\mathbb{P}_{\alpha, \eta}$-name $\dot{\mathcal{U}}_{\alpha, \eta}$ for an ultrafilter such that for all $\beta<\alpha, \Vdash_{\mathbb{P}_{\alpha, \eta}} \dot{\mathcal{U}}_{\beta, \eta} \subseteq \dot{\mathcal{U}}_{\alpha, \eta}$ and every maximal antichain of $\mathbb{M}_{\dot{\mathcal{U}}_{\beta, \eta}}$ from $V_{\beta, \eta}$ is a maximal antichain of $\mathbb{M}_{\mathcal{U}_{\alpha, \eta}}$ (in $V_{\alpha, \eta}$ ) (for the construction of such an ultrafilter in $V^{\mathbb{P}_{\alpha, \eta}}$ see [3], p. 266). If $\operatorname{cf}(\alpha)>\omega$, then let $\dot{\mathcal{U}}_{\alpha, \eta}$ be a $\mathbb{P}_{\alpha, \eta}$-name for $\bigcup_{\beta<\alpha} \mathcal{U}_{\beta, \eta}$. Let $\dot{\mathbb{Q}}_{\alpha, \eta}$ be a $\mathbb{P}_{\alpha, \eta}$-name for $\mathbb{M}_{\dot{\mathcal{U}}_{\alpha, \eta}}$ and let $\mathbb{P}_{\alpha, \zeta}=\mathbb{P}_{\alpha, \eta} * \dot{\mathbb{Q}}_{\alpha, \eta}$. Again by Lemma 13 for all $\beta<\alpha \mathbb{P}_{\beta, \zeta}$ is a complete suborder of $\mathbb{P}_{\alpha, \zeta}$.

If $\zeta \equiv 0 \bmod 2$, then for all $\alpha \leq f(\eta)$ let $\dot{\mathbb{Q}}_{\alpha, \eta}$ be a $\mathbb{P}_{\alpha, \eta}$-name for the trivial poset and for $\alpha>f(\eta)$ let $\dot{\mathbb{Q}}_{\alpha, \eta}$ be a $\mathbb{P}_{\alpha, \eta}$-name for $\mathbb{D}^{V_{f(\eta), \eta}}$. Let $\mathbb{P}_{\alpha, \zeta}=\mathbb{P}_{\alpha, \eta} * \dot{\mathbb{Q}}_{\alpha, \eta}$. If $\alpha<\beta \leq f(\eta)$, then $\mathbb{P}_{\alpha, \zeta}=\mathbb{P}_{\alpha, \eta}, \mathbb{P}_{\beta, \zeta}=\mathbb{P}_{\alpha, \eta}$ and so by the inductive hypothesis $\mathbb{P}_{\alpha, \zeta}$ is a complete suborder of $\mathbb{P}_{\beta, \zeta}$. If $\alpha \leq f(\eta)<$ $\beta$, then $\mathbb{P}_{\alpha, \zeta}=\mathbb{P}_{\alpha, \eta}$ and $\mathbb{P}_{\alpha, \eta}<0 \mathbb{P}_{\beta, \eta}<0 \mathbb{P}_{\beta, \eta} * \dot{\mathbb{Q}}_{\beta, \eta}$. Thus $\mathbb{P}_{\alpha, \zeta}<0 \mathbb{P}_{\beta, \zeta}$. If $f(\eta)<\alpha<\beta$, then again by Lemma $13 \mathbb{P}_{\alpha, \zeta}<0 \mathbb{P}_{\beta, \zeta}$. Furthermore by Lemma 11.(1) $\left(\star^{V_{\alpha, \zeta}, V_{\alpha+1, \zeta}}\right)$ holds for all $\alpha \leq \kappa$.

If $\zeta$ is a limit and for all $\eta<\zeta, \mathbb{P}_{\alpha, \eta}, \dot{\mathbb{Q}}_{\alpha, \eta}$ have been defined, let $\mathbb{P}_{\alpha, \zeta}$ be the finite support iteration of $\left\langle\mathbb{P}_{\alpha, \eta}, \dot{\mathbb{Q}}_{\alpha, \eta}: \eta<\zeta\right\rangle$. By Lemma $10 \mathbb{P}_{\alpha, \zeta}$ is a complete suborder of $\mathbb{P}_{\beta, \zeta}$ and by Lemma 12.(1) $\left(\star_{\mathcal{A}_{\alpha}, A_{\alpha}}^{V_{\alpha, \zeta, \zeta}, V_{\alpha+1, \zeta}}\right)$ holds. 
Remark 14. For all $\alpha<\beta \leq \kappa, \zeta<\eta \leq \lambda \mathbb{P}_{\alpha, \zeta}<\circ \mathbb{P}_{\beta, \eta}$.

Lemma 15. For $\zeta \leq \lambda$ :

(1) for every $p \in \mathbb{P}_{\kappa, \zeta}$ there is $\alpha<\kappa$ such that $p$ belongs to $\mathbb{P}_{\alpha, \zeta}$,

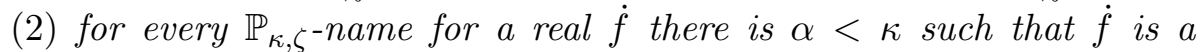
$\mathbb{P}_{\alpha, \zeta}$-name.

Proof. The proof follows just as in the last Lemma of [3], p. 270. For completeness we will give a proof as well. We will prove (1) and (2) simultaneously by induction on $\zeta$. Note that by the ccc property of $\mathbb{P}_{\kappa, \zeta}$ and the fact that $\kappa$ is regular, uncountable, it is clear that (2) follows immediately from (1). If $\zeta=0$ the claim follows from the product property of $\mathbb{P}_{\kappa, 0}$. If $\zeta$ is a limit, $p \in \mathbb{P}_{\kappa, \zeta}$ then since $p$ has a finite support, there is $\eta<\zeta$ such that $p \in \mathbb{P}_{\kappa, \eta}$. By inductive hypothesis there is $\alpha<\eta$ such that $p \in \mathbb{P}_{\alpha, \eta}$ and so in particular $p \in \mathbb{P}_{\alpha, \zeta}$. Let $\zeta=\eta+1$ be a successor. Then $p \in \mathbb{P}_{\kappa, \zeta}$ is of the form $\left(p_{0}, \dot{p}_{1}\right)$ where $p_{0} \in \mathbb{P}_{\kappa, \eta}$ and $\Vdash_{\mathbb{P}_{\kappa, \eta}} \dot{p}_{1} \in \dot{\mathbb{Q}}_{\kappa, \eta}$. If $\zeta \equiv 1 \bmod 2$ then $\dot{p}_{1}$ is of the form $(a, \dot{A})$ where $\Vdash_{\mathbb{P}_{\kappa, \eta}} \dot{A} \in \dot{\mathcal{U}}_{\kappa, \eta}, a \in[\omega]<\omega$. If $\zeta \equiv 0 \bmod 2$, then $\dot{p}_{1}$ is of the form $(s, \dot{f})$ where $\dot{f}$ is a $\mathbb{P}_{\kappa, \eta}$-name for a real, $s \in{ }^{<\omega} \omega$ or $\dot{p}_{1}$ is trivial. In either of the above cases, the inductive hypothesis for (2) implies that there is $\alpha_{1}<\kappa$ such that $\dot{p}_{1}$ is a $\mathbb{P}_{\alpha_{1}, \eta \text {-name. Again the }}$ inductive hypothesis for (1) implies that $p_{0} \in \mathbb{P}_{\alpha_{0}, \eta}$ for some $\alpha_{0}<\kappa$. Then $p=\left(p_{0}, \dot{p}_{1}\right)$ belongs to $\mathbb{P}_{\alpha, \eta}$ where $\alpha=\max \left\{\alpha_{0}, \alpha_{1}\right\}$.

Lemma 16. $V_{\kappa, \lambda} \vDash \mathfrak{b}=\mathfrak{a}=\kappa<\mathfrak{s}=\lambda$.

Proof. The family $\left\{A_{\alpha}\right\}_{\alpha \in \kappa}$ remains a maximal almost disjoint family in $V_{\kappa, \lambda}$. Indeed, otherwise there is a set $B \in V_{\kappa, \lambda} \cap[\omega]^{\omega}$ such that $\forall \alpha<$ $\kappa\left(\left|B \cap A_{\alpha}\right|<\omega\right)$. By Lemma 15 there is $\alpha<\kappa$ such that $B \in V_{\alpha, \lambda} \cap[\omega]^{\omega}$. However $B \notin \mathcal{I}\left(\mathcal{A}_{\alpha}\right)$. On the other hand $\left(\star_{\mathcal{A}_{\alpha}, A_{\alpha+1}}^{V_{\alpha, \lambda}, V_{\alpha+1, \lambda}}\right)$ holds, and so by Lemma $3\left|B \cap A_{\alpha+1}\right|=\omega$ which is a contradiction. Therefore $\mathfrak{a} \leq \kappa$.

Let $\mathcal{B} \subseteq V_{\kappa, \lambda} \cap{ }^{\omega} \omega$ be of cardinality $<\kappa$. Then by Lemma 15 there are $\alpha<\kappa, \zeta<\lambda$ such that $\mathcal{B} \subseteq V_{\alpha, \zeta}$. Since $\{\gamma: f(\gamma)=\alpha\}$ is cofinal in $\lambda$, there is $\zeta^{\prime}>\zeta$ such that $f\left(\zeta^{\prime}\right)=\alpha$. Then $\mathbb{P}_{\alpha+1, \zeta^{\prime}+1}$ adds a real dominating $V_{\alpha, \zeta^{\prime}} \cap{ }^{\omega} \omega$ (and so $V_{\alpha, \zeta} \cap{ }^{\omega} \omega$ since $\left.V_{\alpha, \zeta} \subseteq V_{\alpha, \zeta^{\prime}}\right)$. Thus $\mathcal{B}$ is not unbounded. Therefore $V_{\kappa, \lambda} \Vdash \mathfrak{b} \geq \kappa$. However $\mathfrak{b} \leq \mathfrak{a}$ (see [2]) and so $V_{\kappa, \lambda} \Vdash \mathfrak{b}=\mathfrak{a}=\kappa$.

To see that $V_{\kappa, \lambda} \vDash \mathfrak{s}=\lambda$, note that if $S \subseteq V_{\kappa, \lambda} \cap[\omega]^{\omega}$ is a family of cardinality $<\lambda$, then there is $\zeta<\lambda$ such that $\zeta=\eta+1, \zeta \equiv 1 \bmod 2$ and $S \subseteq V_{\kappa, \eta}$. Then $\mathcal{M}_{\mathcal{U}_{\kappa, \eta}}$ adds a real not split by $S$ and so $S$ is not splitting.

Theorem 17. Let $\kappa<\lambda$ be arbitrary regular uncountable cardinals. Then there is a ccc generic extension in which $\mathfrak{b}=\mathfrak{a}=\kappa<\mathfrak{s}=\lambda$.

\section{The Consistency of $\kappa=\mathfrak{b}<\mathfrak{s}=\mathfrak{a}=\lambda$ Above A Measurable}

Let $\mu$ be a measurable cardinal, $\mathcal{D}$ a $\mu$-complete ultrafilter on $\mu$. Let $\kappa<\lambda$ be regular such that $\mu<\kappa$. For notation regarding ultrapowers of posets and names, see [4] and [10]. In the Lemma below we show that $(\star M, N, c)$ is preserved under ultrapowers.

Lemma 18. Let $\mathbb{P}<0 \mathbb{Q}, c \in V^{\mathbb{Q}}$ such that for all $f \in V^{\mathbb{P}} \cap{ }^{\omega} \omega$, $\Vdash_{\mathbb{P}} c \mathbb{Z}^{*} f$. Let $\mathbb{Q}^{\prime}=\mathbb{Q}^{\mu} / \mathcal{D}, \mathbb{P}^{\prime}=\mathbb{P}^{\mu} / \mathcal{D}, f \in V^{\mathbb{P}^{\prime}} \cap \omega \omega$. Then $\Vdash_{\mathbb{Q}^{\prime}} c \not^{*} f$. 
Proof. Suppose not. Thus there is a $\mathbb{P}^{\prime}$ name for a real $\dot{f}$ and $[q] \in \mathbb{Q}^{\prime}$ such that for some $k \in \omega$, for all $i \geq k,[q] \Vdash \dot{c}(i) \leq \dot{f}(i)$. Note that $\dot{f}$ is determined by maximal antichains $\left\{\left[p_{n, i}\right]\right\}_{n, i \in \omega}$ and $\left\{k_{n, i}\right\}_{n, i \in \omega} \subseteq \omega$ such that for all $n, i\left[p_{n, i}\right] \Vdash_{\mathbb{P}^{\prime}} \dot{f}(i)=k_{n, i}$. Furthermore we can assume (see [4]) that for all $\alpha \in \mu$ there are maximal antichains $\left\{p_{n, i}^{\alpha}\right\}_{n, i \in \omega}$ in $\mathbb{P}$, such that $\left[p_{n, i}\right]=\left\langle p_{n, i}^{\alpha}: \alpha<\mu\right\rangle / \mathcal{D}$, and a $\mathbb{P}$-name for a real $\dot{f}^{\alpha}$ such that $p_{n, i}^{\alpha} \Vdash_{\mathbb{P}}$ $\dot{f}^{\alpha}(i)=k_{n, i}$. By elementarity, $A=\left\{\alpha: q(\alpha) \Vdash_{\mathbb{Q}} \dot{c}(i) \leq \dot{f}^{\alpha}(i)\right\}$ is in $\mathcal{D}$, and so in particular $A$ is non-empty. Here we identify $\dot{c}$ with its ultrapower. Let $\alpha \in A$. Then $\dot{f}^{\alpha}$ is a $\mathbb{P}$-name and for all $i \geq k, q(\alpha) \Vdash_{\mathbb{Q}} \dot{c}(i) \leq f^{\alpha}(i)$, which is a contradiction.

Let $f:\{\eta<\lambda: \eta \equiv 1 \bmod 3\} \rightarrow \kappa$ be an onto mapping such that for all $\alpha<\kappa, f^{-1}(\alpha)$ is cofinal in $\lambda$. Similarly to the construction from the previous section, recursively define a system of finite support iterations $\left\langle\left\langle\mathbb{P}_{\alpha, \zeta}: \alpha \leq \kappa, \zeta \leq \lambda\right\rangle,\left\langle\dot{\mathbb{Q}}_{\alpha, \zeta}: \alpha \leq \kappa, \zeta<\lambda\right\rangle\right\rangle$ so that properties $(1)-(5)$, as well as $(a)-(b)$ below hold. For all $\alpha, \zeta$ let $V_{\alpha, \zeta}=V^{\mathbb{P}_{\alpha, \zeta}}$.

(1) If $\zeta=0$, then for all $\alpha \leq \kappa$, let $\mathbb{P}_{\alpha, 0}$ be the forcing notion for adding $\alpha$ many Cohen reals, $\left\{c_{\gamma}\right\}_{\gamma<\alpha}$.

(2) If $\zeta=\eta+1, \zeta \equiv 1 \bmod 3$, then $\Vdash_{\mathbb{P}_{\alpha, \eta}} \dot{\mathbb{Q}}_{\alpha, \eta}=\mathbb{M}_{\dot{\mathcal{U}}_{\alpha, \eta}}$ where $\dot{\mathcal{U}}_{\alpha, \eta}$ is a $\mathbb{P}_{\alpha, \eta}$-name for an ultrafilter and for all $\alpha<\beta \leq \kappa, \Vdash_{\mathbb{P}_{\beta, \eta}} \dot{\mathcal{U}}_{\alpha, \eta} \subseteq \dot{\mathcal{U}}_{\beta, \eta}$.

(3) If $\zeta=\eta+1, \zeta \equiv 2 \bmod 3$, then if $\alpha \leq f(\eta)$, $\dot{\mathbb{Q}}_{\alpha, \eta}$ is a $\mathbb{P}_{\alpha, \eta}$-name for the trivial forcing notions; if $\alpha>f(\eta)$ then $\dot{\mathbb{Q}}_{\alpha, \eta}$ is a $\mathbb{P}_{\alpha, \eta}$-name for $\mathbb{D}^{V_{f(\eta), \eta}}$.

(4) If $\zeta=\eta+1, \zeta \equiv 0 \bmod 3$, then for every $\alpha \leq \kappa$ let $\dot{\mathbb{Q}}_{\alpha, \eta}$ be a $\mathbb{P}_{\alpha, \eta}$-name for the quotient poset of $\left(\left(\mathbb{P}_{\alpha, \eta}\right)^{\mu}\right) / \mathcal{D}$ and $\mathbb{P}_{\alpha, \eta}$.

(5) If $\zeta$ is a limit, $\mathbb{P}_{\alpha, \zeta}$ is the finite support iteration of $\left\langle\mathbb{P}_{\alpha, \eta}, \dot{\mathbb{Q}}_{\alpha, \eta}: \eta<\zeta\right\rangle$.

Furthermore the construction will satisfy the following two properties:

(a) $\forall \zeta \leq \lambda \forall \alpha<\beta \leq \kappa, \mathbb{P}_{\alpha, \zeta}$ is a complete suborder of $\mathbb{P}_{\beta, \zeta}$.

(b) $\forall \zeta \leq \lambda \forall \alpha<\kappa\left(\star V_{\alpha, \zeta}, V_{\alpha+1, \zeta}, c_{\alpha+1}\right)$ holds.

Proceed by induction on $\zeta$. If $\zeta=0$, then for all $\alpha \leq \kappa$ let $\mathbb{P}_{\alpha, 0}$ be the forcing notion for adding $\alpha$ many Cohen reals, $\left\{c_{\gamma}\right\}_{\gamma<\alpha}$. The properties of Cohen forcing imply that $(a)$ and $(b)$ above hold. Let $\zeta=\eta+1$ be a successor ordinal and suppose that for all $\alpha \leq \kappa, \mathbb{P}_{\alpha, \eta}$ has been defined so that the relevant properties $(a)$ and $(b)$ above hold.

If $\zeta \equiv 1 \bmod 3$ define $\dot{\mathbb{Q}}_{\alpha, \eta}$ by induction on $\alpha \leq \kappa$ as follows. If $\alpha=0$ let $\dot{\mathcal{U}}_{0, \eta}$ be a $\mathbb{P}_{0, \eta}$-name for an ultrafilter, $\dot{\mathbb{Q}}_{0, \eta}$ a $\mathbb{P}_{0, \eta}$-name for $\mathbb{M}_{\dot{\mathcal{U}}_{0, \eta}}$ and let $\mathbb{P}_{0, \zeta}=\mathbb{P}_{0, \eta} * \dot{\mathbb{Q}}_{0, \eta}$. If $\alpha=\beta+1$ and $\dot{\mathcal{U}}_{\beta, \eta}$ has been defined, by the inductive hypothesis and Lemma 6 there is a $\mathbb{P}_{\alpha, \eta}$-name $\dot{\mathcal{U}}_{\alpha, \eta}$ for an ultrafilter such that $\Vdash_{\mathbb{P}_{\alpha, \eta}} \dot{\mathcal{U}}_{\beta, \eta} \subseteq \dot{\mathcal{U}}_{\alpha, \eta}$, every maximal antichain of $\mathbb{M}_{\dot{\mathcal{U}}_{\beta, \eta}}$ in $V_{\beta, \eta}$ is a maximal

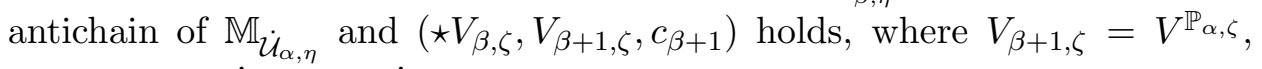
$\mathbb{P}_{\alpha, \zeta}=\mathbb{P}_{\alpha, \eta} * \dot{\mathbb{Q}}_{\alpha, \eta}$ and $\dot{\mathbb{Q}}_{\alpha, \eta}$ is a $\mathbb{P}_{\alpha, \eta}$-name for $\mathbb{M}_{\dot{\mathcal{U}}_{\alpha, \eta}}$. By Lemma $13 \mathbb{P}_{\beta, \zeta}=$ $\mathbb{P}_{\beta, \eta} * \mathbb{M}_{\dot{\mathcal{U}}_{\beta, \eta}}$ is a complete suborder of $\mathbb{P}_{\alpha, \zeta}$. If $\alpha$ is a limit ordinal and for all $\beta<\alpha, \dot{\mathcal{U}}_{\beta, \eta}$ has been defined, proceed as in the limit case for $\alpha, \zeta$ successor, 
odd ordinal, in the construction of the system of finite support iterations from the previous section.

If $\zeta \equiv 2 \bmod 3$ then for all $\alpha \leq f(\eta)$ let $\dot{\mathbb{Q}}_{\alpha, \eta}$ be a $\mathbb{P}_{\alpha, \eta}$-name for the trivial poset and for $\alpha>f(\eta)$ let $\dot{\mathbb{Q}}_{\alpha, \eta}$ be a $\mathbb{P}_{\alpha, \eta}$-name for $\mathbb{D}^{V_{f(\eta), \eta}}$. Let $\mathbb{P}_{\alpha, \zeta}=\mathbb{P}_{\alpha, \eta} * \dot{\mathbb{Q}}_{\alpha, \eta}$. Property $(a)$ can be established for $\zeta$ and $\alpha \leq \kappa$ just as in the successor, even case in the construction from the previous section. The inductive hypothesis and Lemma 11.(2) imply that for $\zeta$ and $\alpha \leq \kappa$, property $(b)$ holds as well.

If $\zeta \equiv 0 \bmod 3$ then for all $\alpha \leq \kappa$ let $\dot{\mathbb{Q}}_{\alpha, \eta}$ be a $\mathbb{P}_{\alpha, \eta}$-name for the quotient poset of $\left(\mathbb{P}_{\alpha, \eta}^{\mu}\right) / \mathcal{D}$ and $\mathbb{P}_{\alpha, \eta}$. Let $\mathbb{P}_{\alpha, \zeta}=\mathbb{P}_{\alpha, \eta} * \dot{\mathbb{Q}}_{\alpha, \eta}$. Then by the inductive hypothesis and Lemma 5 of [4] for all $\alpha<\beta \leq \kappa \mathbb{P}_{\alpha, \zeta}$ is a complete suborder of $\mathbb{P}_{\beta, \zeta}$. By the inductive hypothesis and Lemma 18 for all $\alpha<\kappa$ $\left(\star V_{\alpha, \zeta}, V_{\alpha+1, \zeta}, c_{\alpha+1}\right)$ holds.

If $\zeta$ is a limit and for all $\eta<\zeta, \mathbb{P}_{\alpha, \eta}$ and $\dot{\mathbb{Q}}_{\alpha, \eta}$ have been defined, then let $\mathbb{P}_{\alpha, \zeta}$ be the finite support iteration of $\left\langle\mathbb{P}_{\alpha, \eta}, \dot{\mathbb{Q}}_{\alpha, \eta}: \eta\langle\zeta\rangle\right.$. By Lemma 10 for all $\alpha<\beta \leq \kappa \mathbb{P}_{\alpha, \zeta}$ is a complete suborder of $\mathbb{P}_{\beta, \zeta}$ and by Lemma 12.(2) $\left(\star V_{\alpha, \zeta}, V_{\alpha+1, \zeta}, c_{\alpha+1}\right)$ holds for all $\alpha<\kappa$.

Lemma 19. For $\zeta \leq \lambda$ :

(1) for every $p \in \mathbb{P}_{\kappa, \zeta}$ there is $\alpha<\kappa$ such that $p$ belongs to $\mathbb{P}_{\alpha, \zeta}$,

(2) for every $\mathbb{P}_{\kappa, \zeta}$-name for a real $\dot{f}$ there is $\alpha<\kappa$ such that $\dot{f}$ is a $\mathbb{P}_{\alpha, \zeta}$-name.

Proof. If $\zeta$ is a limit, proceed as in the limit case of Lemma 15. If $\zeta=\eta+1$ is a successor and $\zeta \equiv 1 \bmod 3$ or $\zeta \equiv 2 \bmod 3$ the proof follows as in the successor case of Lemma 15. Let $\zeta \equiv 0 \bmod 3$ and let $p \in\left(\mathbb{P}_{\kappa, \eta}\right)^{\mu} / \mathcal{D}$. Then $p=[f]=\langle f(\gamma): \gamma<\mu\rangle / \mathcal{D}$ where $f(\gamma) \in \mathbb{P}_{\kappa, \eta}$ for $\gamma<\mu$. By the inductive hypothesis and $\kappa=\operatorname{cf}(\kappa)>\mu$, there is $\alpha<\kappa$ such that $f(\gamma) \in \mathbb{P}_{\alpha, \eta}$ for all $\gamma<\mu$ and so $p=[f]$ belongs to $\left(\mathbb{P}_{\alpha, \eta}\right)^{\mu} / \mathcal{D}=\mathbb{P}_{\alpha, \zeta}$.

Lemma 20. $V_{\kappa, \lambda} \vDash \mathfrak{b}=\kappa<\mathfrak{s}=\mathfrak{a}=\lambda$,

Proof. Let $\dot{f} \in V_{\kappa, \lambda} \cap{ }^{\omega} \omega$. Then there are $\zeta<\lambda, \alpha<\kappa$ such that $f \in$ $V_{\alpha, \zeta} \cap{ }^{\omega} \omega$. Since $\left(\star V_{\alpha, \zeta} V_{\alpha+1, \zeta} c_{\alpha+1}\right)$ holds, $V_{\alpha+1, \zeta} \vDash c_{\alpha+1} \mathbb{Z}^{*} f$ and so $V_{\kappa, \lambda} \vDash c_{\alpha+1} \mathbb{Z}^{*} f$. Therefore $\left\{c_{\alpha+1}\right\}_{\alpha<\kappa}$ is unbounded in $V_{\kappa, \lambda}$. If $\mathcal{B} \subseteq$ $V_{\kappa, \lambda} \cap{ }^{\omega} \omega$ is a family of reals of cardinality $<\kappa$, then there are $\alpha<\kappa, \zeta<\lambda$ such that $\mathcal{B} \subseteq V_{\alpha, \zeta}$. Since $\{\gamma: f(\gamma)=\alpha\}$ is cofinal in $\lambda$, there is $\zeta^{\prime}>\zeta$ such that $f\left(\zeta^{\prime}\right)=\alpha$. Therefore $(\mathfrak{b}=\kappa)^{V_{\kappa, \lambda}}$.

Since $\mathfrak{a} \geq \mathfrak{b}$, we have $V_{\kappa, \lambda} \vDash \mathfrak{a} \geq \kappa$. Let $\mathcal{A} \subseteq V_{\kappa, \lambda} \cap[\omega]^{\omega}$ be an almost disjoint family of cardinality $\nu$ where $\kappa \leq \nu<\lambda$. Then there is $\zeta<\lambda$ such that $\zeta=\eta+1, \zeta \equiv 0 \bmod 3$ and $\mathcal{A} \subseteq V_{\kappa, \eta}$. Then by Lemma 4 of [4], in $V_{\kappa, \zeta}$ there is a real which has a finite intersection with all elements of $\mathcal{A}$ and so $\mathcal{A}$ is not maximal. Therefore $(\mathfrak{a}=\mathfrak{c}=\lambda)^{V_{\kappa, \lambda}}$.

To see that $(\mathfrak{s}=\lambda)^{V_{\kappa, \lambda}}$ note that if $S \subseteq V_{\kappa, \lambda} \cap[\omega]^{\omega},|S|<\lambda$, then there is $\zeta<\lambda, \zeta=\eta+1, \zeta \equiv 1 \bmod 3$ such that $S \subseteq V_{\kappa, \eta}$. Then in $V_{\kappa, \zeta}$ there is a real which is not split by $S$ (added by $\mathbb{M}_{\mathcal{U}_{\kappa, \eta}}$ ) and so $S$ is not splitting.

Thus we obtain the following theorem.

Theorem 21. Let $\mu$ be a measurable cardinal, $\kappa<\lambda$ regular such that $\mu<\kappa$. Then there is a ccc generic extension in which $\mathfrak{b}=\kappa<\mathfrak{s}=\mathfrak{a}=\lambda$. 


\section{Comments And QUeStions}

The converse consistency $\kappa=\mathfrak{s}<\mathfrak{b}=\mathfrak{a}=\lambda$ is well-known and standard. However of interest remain the following questions:

(1) Is it relatively consistent that $\mathfrak{b}<\mathfrak{a}<\mathfrak{s}$ ?

(2) Is it relatively consistent that $\mathfrak{b}<\mathfrak{s}<\mathfrak{a}$ ?

(3) Is it relatively consistent that $\mathfrak{b}<\mathfrak{s}=\mathfrak{a}$ without assuming a measurable?

\section{REFERENCES}

[1] J. Baumgartner, P. Dordal Adjoining dominating functions, The Journal of Symbolic Logic 50(1985), 94 - 101.

[2] A. Blass Combinatorial Cardinal Characteristics of the Continuum, for the Handbook of Set-Theory.

[3] A. Blass and S. Shelah Ultrafilters with Small Generating Sets, Israel Journal of Mathematics 65(1984), 259-271.

[4] J. Brendle Mad families and Ultrafilters Acta Universitatis Carolinae. Mathematica et Physica 49(2007), 19-35.

[5] J. Brendle Mob families and mad families Arch. Math. Logic 37(1998), 183-197.

[6] V. Fischer The consistency of arbitrarily large spread between the bounding and the splitting numbers, doctoral dissertation, York University, 2008.

[7] V. Fischer, J. Steprāns The consistency of $\mathfrak{b}=\kappa<\mathfrak{a}=\kappa^{+}$Fundamenta Mathematicae 201(2008), 283 - 293.

[8] S. Hechler Short complete nested sequences in $\beta \mathbb{N} / \mathbb{N}$ and small maximal almost-disjoint families General Topology and its Applications 2(1972), 139-149.

[9] S. Shelah On cardinal invariants of the continuum Contemporary Mathematics 31(1984), 184-207.

[10] S. Shelah Two cardinal invariants of the coninuum $(\mathfrak{d}<\mathfrak{a})$ and FS linearly ordered iterated forcing Acta Math. 192(2004), 187-223.

Graduate School of Engineering, Kobe University, Rokko-dai 1-1, Nada-Ku, KOBE 657-8501, JAPAN

E-mail address: brendle@kurt.scitec.kobe-u.ac.jp

Kurt Gödel Research Center, University of Vienna, Währinger Strasse 25, 1090 Vienna, Austria

E-mail address: vfischer@logic.univie.ac.at 